\title{
Comparison of the Prognosis of Decompensated Cirrhosis in Patients with and Without Eradication of Hepatitis C Virus
}

\author{
Takashi Kumada (D) - Hidenori Toyoda $\cdot$ Satoshi Yasuda • \\ Toshifumi Tada · Junko Tanaka · Kazuaki Chayama · Philip J. Johnson • \\ William L. Irving
}

Received: January 29, 2021 / Accepted: March 24, 2021 / Published online: April 21, 2021

(c) The Author(s) 2021

\section{ABSTRACT}

Introduction: In patients with hepatitis $C$ virus (HCV) infection and decompensated cirrhosis (DC), it is uncertain whether viral clearance is clinically meaningful and whether it decreases liver-related and non-liver-related mortality. The aim of this study was to assess whether viral

Supplementary Information The online version contains supplementary material available at https:// doi.org/10.1007/s40121-021-00441-7.

T. Kumada $(\square)$

Department of Nursing, Faculty of Nursing, Gifu Kyoritsu University, 5-50, Kitagata-cho, Ogaki-shi, Gifu-ken, Ogaki, Gifu 503-8550, Japan

e-mail: takashi.kumada@gmail.com

H. Toyoda $\cdot$ S. Yasuda $\cdot$ T. Tada

Department of Gastroenterology and Hepatology,

Ogaki Municipal Hospital, Ogaki, Gifu, Japan

H. Toyoda

e-mail: tkumada@he.mirai.ne.jp

S. Yasuda

e-mail: satoshi.yasuda.1982@gmail.com

T. Tada

e-mail: tadat0627@gmail.com

J. Tanaka

Department of Epidemiology, Infectious Disease Control, and Prevention, Hiroshima University Institute of Biomedical and Health Sciences,

Hiroshima, Japan

e-mail: jun-tanaka@hiroshima-u.ac.jp eradication reduced liver-related and non-liverrelated mortality in patients with $\mathrm{HCV}$ infection and DC.

Methods: To clarify the impact of viral eradication on liver-related and non-liver-related mortality, 364 patients with DC who received direct-acting antivirals (DAAs) and achieved sustained virological response (SVR) in the UK (DAA group) were compared with 249 patients with DC who did not receive DAAs and who underwent symptomatic treatment in Japan (non-DAA group). Propensity score matching and inverse probability weighting (IPW) were

\section{K. Chayama}

Department of Gastroenterology and Metabolism, Institute of Biomedical and Health Sciences, Hiroshima University Hospital, Hiroshima, Japan e-mail: chayama@mba.ocn.ne.jp

\section{P. J. Johnson}

Department of Molecular and Clinical Cancer Medicine, University of Liverpool, Liverpool, UK e-mail: Philip.Johnson@liverpool.ac.uk

\section{W. L. Irving}

NIHR Nottingham Biomedical Research Centre, Nottingham University Hospitals NHS Trust, The University of Nottingham, Nottingham, UK e-mail: Will.Irving@nottingham.ac.uk 
performed to adjust the baseline characteristics in the DAA and non-DAA groups. Liver-related and non-liver-related mortality were analyzed using the competing risks IPW cumulative incidence functions estimator.

Results: The cumulative all-cause mortality rate in the DAA group was significantly lower than that in the non-DAA group $(p<0.0001$, IPW-adjusted log-rank test). The cumulative incidence rates of both liver-related and nonliver-related mortality were significantly lower in the DAA group than those in the non-DAA group ( $p<0.0001$ for both).

Conclusion: DAA-mediated viral eradication reduced not only liver-related mortality but also non-liver-related mortality in patients with HCV infection and DC.

Keywords: Hepatitis C virus (HCV); Decompensated cirrhosis; Direct-acting antiviral (DAA); Sustained virological response (SVR); Hepatocarcinogenesis; Liver-related mortality; Non-liver-related mortality

\section{Key Summary Points}

\section{Why carry out this study?}

It is uncertain whether viral clearance is meaningful and reduces liver-related and non-liver-related mortality in patients with hepatitis $\mathrm{C}$ virus (HCV) infection and decompensated cirrhosis (DC).

The aim of this study was to assess whether viral eradication reduced liverrelated and non-liver-related mortality in patients with HCV infection and DC.

\section{What was learned from the study?}

Eradication of HCV reduced not only liverrelated mortality but also non-liverrelated mortality in patients with hepatitis $C$ virus infection and DC.

Patients with HCV infection and DC should be actively treated with antiviral therapy whenever possible, regardless of liver function.

\section{DIGITAL FEATURES}

This article is published with digital features, including a summary slide, to facilitate understanding of the article. To view digital features for this article go to https://doi.org/10.6084/ m9.figshare.14258693.

\section{INTRODUCTION}

One of the most severe sequelae of chronic infection with the hepatitis $\mathrm{C}$ virus $(\mathrm{HCV})$ is decompensated cirrhosis (DC) [1, 2]. Patients with DC have a poor prognosis and treatment options remain limited. Complications of DC include jaundice, variceal hemorrhage, ascites, and encephalopathy. Interferon-based treatment has not been recommended for HCV-infected patients with DC because of the severity of adverse effects in this population [3, 4]. However, the introduction of oral direct-acting antiviral (DAA)-based HCV therapy has dramatically increased the number of patients who are eligible for antiviral therapy, and high response rates can be achieved with various combination treatment regimens $[5,6]$.

Viral eradication in patients with $\mathrm{HCV}$ infection and chronic hepatitis or compensated cirrhosis reduces all-cause mortality, including liver-related and non-liver-related mortality [7-9]. In a previous study of patients with DC, viral eradication was associated with a rapid improvement in liver function and complications $[10,11]$. Two previous studies in patients with DC enrolled in a UK hepatitis $C$ registry, HCV Research UK, had a follow-up period of less than 12 months $[12,13]$. Therefore, in patients with HCV infection and DC, it is uncertain whether viral clearance is meaningful and whether it reduces liver-related and non-liverrelated mortality.

The aim of this study was to assess whether viral eradication decreased liver-related and non-liver-related mortality in patients with HCV infection and DC. The treatment cohort, which was enrolled in the HCV Research UK registry, received DAA therapy, achieved sustained virological response (SVR), and was then prospectively followed for 3 years. The no- 
treatment cohort was enrolled at one hospital in Japan and included only patients who did not receive DAAs.

\section{METHODS}

\section{Ethics}

Ethics approval for HCV Research UK was given by the National Research Ethics Service (NRES) Committee East Midlands-Derby 1 (Research Ethics Committee reference 11/EM/0314), and the proposal for the analysis reported herein was accepted by the HCV Research UK Tissue and Data Access committee in January 2019 (Request No. TR000444). Informed consent was obtained from each patient included in the UK group. The requirement for consent to participate in the Japanese group was waived by the ethics committee because of the retrospective nature of the study. The study protocol was also approved by the institutional review board of Ogaki Municipal Hospital in March 2019 and was conducted in compliance with the Helsinki Declaration.

\section{Patients}

Between May 2014 and September 2015, 653 patients with a history of DC who received DAA therapy were enrolled in the HCV Research UK registry for prospective data collection. Inclusion criteria for DC comprised ascites, variceal bleeding, jaundice, or hepatic encephalopathy (past or current). Treatment involved mainly ledipasvir/sofosbuvir, sofosbuvir/daclatasvir, or other regimens with or without ribavirin for a total of 12 weeks. The overall SVR rates of intention-to treat was $86.2 \%(563 / 653)$. Of the 653 patients, 364 patients were analyzed as the "DAA group." (Fig. 1).

As a control, an untreated cohort of patients with decompensated HCV cirrhosis who did not receive DAA therapy and who underwent symptomatic treatment was studied at Ogaki Municipal Hospital in Japan between 2000 and 2007. Of 2981 consecutive patients whose data were collected retrospectively, 249 patients were enrolled as the control "non-DAA group" (Fig. 1).

Observation was started on the first day of DAA treatment in the DAA group and on the day of DC diagnosis in the non-DAA group. Observation was terminated on the day of death or the last visit in both groups.

At the start of follow-up, alanine aminotransferase (ALT), aspartate aminotransferase (AST), platelet count, albumin, total bilirubin, prothrombin time-international normalized ratio (PT-INR), creatinine, and estimated glomerular filtration rate (eGFR) [14] were measured in all patients. The FIB-4 score was calculated at the start of follow-up according to Vallet-Pichard et al. [15]. Patients with a FIB-4 score greater than 3.25 were defined as having extensive fibrosis [16]. In addition, the Child-Pugh classification, Child-Pugh score, model for end-stage liver disease (MELD) score, and albumin-bilirubin (ALBI) grade were used as indicators of liver function [3]. The ALBI grade was calculated according to Johnson et al. [17]. The continuous linear predictor was further categorized into three different grades for prognostic stratification purposes, as previously described: grade 1 (less than -2.60 ), grade 2 (between -2.60 and -1.39 ), and grade 3 (above - 1.39) [17]. SVR was defined as undetectable serum HCV RNA at 12 weeks after the end of treatment. Diabetes mellitus (DM) was diagnosed on the basis of the criteria of the American Diabetes Association [18]. Excessive alcohol consumption was defined as more than $80 \mathrm{~g}$ of ethanol per day.

In this study, causes of death were divided into liver-related disease, which included HCC, liver failure, and variceal bleeding, and nonliver-related disease. Causes of death in the UK were determined from the descriptions in the HCV Research UK database. Causes of death of patients who died at Ogaki Municipal Hospital in Japan were retrospectively identified by reviewing medical records; for patients who died elsewhere, for example in other hospitals, hospices, or their own homes, information regarding cause of death was obtained from the attending physician or the family physician. 
2,981 consecutive patients treated for $\mathrm{HCV}$ infection between 2000 and 2017 at Ogaki

Municipal Hospital in Japan.

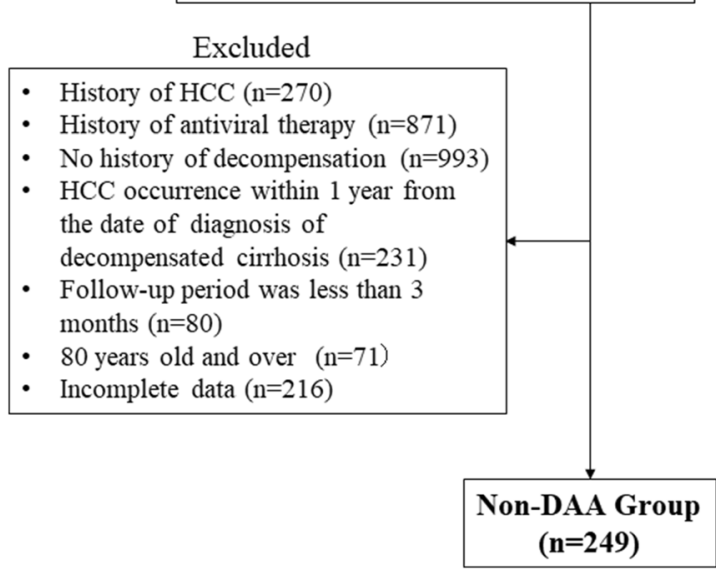

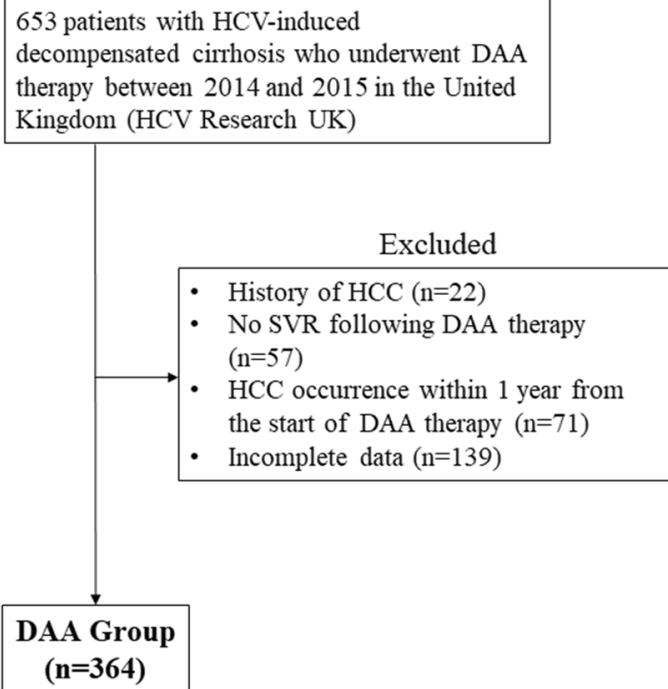

653 patients with HCV-induced

cirrhosis who underwent

Kingdom (HCV Research UK)

$(n=364)$

Fig. 1 Flowchart of the patient selection process. HCV hepatitis C virus, DAA direct-acting antiviral, SVR sustained virological response, HCC hepatocellular carcinoma

\section{Statistical Analysis}

Continuous variables were expressed as medians (the first to third quartiles). The Mann-Whitney $U$ test was used to assess continuous variables. Fisher's exact test was used to evaluate categorical variables.

Multivariate Cox proportional hazards models were used to analyze factors related to hepatocarcinogenesis and all-cause mortality. Fine and Gray proportional hazards models for the subdistribution of a competing risk were used to analyze factors related to liver-related, non-liver-related mortality, and transplantation [19]. Liver transplantation was not included in this analysis because it was very small in the non-DAA group (Table 1). The analyzed factors were age, gender, body mass index (BMI), alcohol abuse (presence or absence), DM (presence or absence), ascites (presence or absence), hepatic encephalopathy (presence or absence), antiviral therapy (DAA vs. non-DAA), AST, ALT, total bilirubin, albumin, platelet count, PT-INR, and creatinine.

In this study, we performed propensity score matching since the baseline characteristics were different between the DAA and non-DAA group. The multivariate logistic regression model for propensity score matching comprised the following parameters: age, gender, BMI, alcohol abuse, DM, ascites, hepatic encephalopathy, AST, ALT, total bilirubin, albumin, platelet count, PT-INR, and creatinine. Weights were truncated at the 99th percentile to prevent sparse data (weights could range from 0 to infinity, and those that were extreme outliers were deleted). After the propensity score was established, we applied inverse probability weighting (IPW), which was defined as 1/ (propensity score) for the DAA group and 1/ ( 1 - propensity score) for the non-DAA group [20]. We estimated the rates of hepatocarcinogenesis and all-cause mortality using IPW as a treatment effect. Differences were tested using the IPW-adjusted log-rank test. In the presence of competing events, including liver-related and non-liver-related mortality, the naïve Kaplan-Meier (KM) estimator derived by censoring competing events does not correctly estimate the cumulative incidence function (CIF) for the events of interest (referred to as the KM CIF estimator). To appropriately account for the competing events, IPW-adjusted CIFs (referred to as the competing risks CIF estimators) were calculated according to the report of Bolch et al. [21].

Statistical significance was defined as $p<0.05$. All statistical analyses were performed 
Table 1 Comparison of baseline characteristics between the DAA and non-DAA groups

\begin{tabular}{|c|c|c|c|}
\hline & DAA group $(n=364)$ & Non-DAA group $(n=249)$ & $p$ value \\
\hline Age (years) & $54(48-59)$ & $70(64-74)$ & $<0.001$ \\
\hline Gender (male) & $262(72.0)$ & $136(54.6)$ & $<0.001$ \\
\hline \multicolumn{4}{|l|}{ Race } \\
\hline Asian & $36(9.69)$ & $248(100.0)$ & $<0.001$ \\
\hline White & $304(83.5)$ & $0(0.0)$ & \\
\hline Black & $8(2.2)$ & $0(0.0)$ & \\
\hline Others & $16(4.4)$ & $0(0.0)$ & \\
\hline Diabetes mellitus & $74(20.3)$ & $126(50.2)$ & $<0.001$ \\
\hline Alcohol abuse & $181(49.7)$ & $17(6.8)$ & $<0.001$ \\
\hline Smoking & $255(75.0)$ & $57(31.8)$ & $<0.001$ \\
\hline Hepatic encephalopathy & $121(33.2)$ & $31(12.4)$ & $<0.001$ \\
\hline Ascites & $271(74.5)$ & $228(91.6)$ & $<0.001$ \\
\hline \multicolumn{4}{|l|}{ Child-Pugh classification } \\
\hline A & $50(13.7)$ & $36(14.5)$ & 0.292 \\
\hline $\mathrm{B}$ & $276(75.8)$ & $177(71.1)$ & \\
\hline $\mathrm{C}$ & $38(10.4)$ & $36(14.5)$ & \\
\hline Child-Pugh score & $7(7-8)$ & $8(7-9)$ & 0.001 \\
\hline MELD score & $11.5(9.2-13.9)$ & $10.3(7.5-12.9)$ & 0.001 \\
\hline BMI $\left(\mathrm{kg} / \mathrm{m}^{2}\right)$ & $27.0(24.0-30.7)$ & $22.1(20.3-24.7)$ & $<0.001$ \\
\hline \multicolumn{4}{|l|}{ Genotype $^{a}$} \\
\hline 1 & $193(53.5)$ & $152(74.1)$ & $<0.001$ \\
\hline 2 & $16(4.4)$ & $53(25.9)$ & \\
\hline 3 & $129(35.7)$ & $0(0.0)$ & \\
\hline 4 & $23(6.4)$ & $0(0.0)$ & \\
\hline $\operatorname{ALT}(\mathrm{U} / \mathrm{L})$ & $52(34-79)$ & $35(23-55)$ & $<0.001$ \\
\hline $\operatorname{AST}(\mathrm{U} / \mathrm{L})$ & $80(55-117)$ & $52(36-77)$ & $<0.001$ \\
\hline Platelet count $\left(\times 10^{4} / \mu \mathrm{L}\right)$ & $7.4(5.6-10.4)$ & $10.7(7.1-16.0)$ & $<0.001$ \\
\hline FIB-4 score & $7.87(5.03-12.79)$ & $5.82(3.38-9.36)$ & $<0.001$ \\
\hline$\leq 3.25$ & $26(7.1)$ & $61(24.5)$ & $<0.001$ \\
\hline$>3.25$ & $338(92.9)$ & $188(75.5)$ & \\
\hline Albumin (g/dL) & $3.1(2.8-3.5)$ & $3.0(2.7-3.5)$ & 0.017 \\
\hline Total bilirubin $(\mathrm{mg} / \mathrm{dL})$ & $1.6(1.0-2.3)$ & $1.1(0.6-1.9)$ & $<0.001$ \\
\hline ALBI score & $-1.74(-2.14$ to -1.39$)$ & $-1.77(-2.20$ to -1.37$)$ & 0.523 \\
\hline
\end{tabular}


Table 1 continued

\begin{tabular}{|c|c|c|c|}
\hline & DAA group $(n=364)$ & Non-DAA group $(n=249)$ & $p$ value \\
\hline \multicolumn{4}{|l|}{ ALBI grade } \\
\hline 1 & $29(8.0)$ & $34(10.6)$ & 0.890 \\
\hline 2 & $244(67.0)$ & $207(64.7)$ & \\
\hline 3 & $91(25.0)$ & $79(24.7)$ & \\
\hline Creatinine $(\mathrm{mg} / \mathrm{dL})$ & $0.76(0.66-0.93)$ & $0.70(0.60-1.01)$ & 0.082 \\
\hline eGFR $\left(\mathrm{mL} / \mathrm{min} / 1.73 \mathrm{~m}^{2}\right)$ & $76.3(60.1-93.4)$ & $73.2(53.7-89.7)$ & 0.001 \\
\hline PT-INR & $1.30(1.10-1.40)$ & $1.13(0.99-1.30)$ & $<0.001$ \\
\hline Liver transplantation ${ }^{\mathrm{b}}$ & $38(10.4)$ & $2(0.6)$ & $<0.001$ \\
\hline Occurrence of HCC & $15(4.1)$ & $49(19.7)$ & $<0.001$ \\
\hline Deaths & $48(23.5)$ & $156(76.5)$ & $<0.001$ \\
\hline Liver-related death & $19(39.6)$ & $79(50.6)$ & 0.191 \\
\hline Non-liver-related death & $29(60.4)$ & $77(49.4)$ & \\
\hline Observation period (years) & $1.75(0.71-3.05)$ & $2.96(1.07-5.79)$ & $<0.001$ \\
\hline
\end{tabular}

Continuous values are expressed as medians (the first to third quartiles)

$D A A$ direct-acting antiviral, $M E L D$ model for end-stage liver disease, $B M I$ body mass index, $A L T$ alanine aminotransferase, $A S T$ aspartate aminotransferase, $A L B I$ albumin-bilirubin, $e G F R$ estimated glomerular filtration rate, $P T-I N R$ prothrombin time-international normalized ratio, $H C C$ hepatocellular carcinoma

${ }^{a}$ Data missing for three patients in DAA group and 44 in non-DAA group

b Thirty patients received DAA therapy before liver transplantation

with EZR (Saitama Medical Center, Jichi Medical University, Saitama, Japan), which is a graphical user interface for $\mathrm{R}$ (R Foundation for Statistical Computing, Vienna, Austria) [22]. More precisely, it is a modified version of $\mathrm{R}$ commander designed to add statistical functions frequently used in biostatistics.

\section{RESULTS}

\section{Differences in Baseline Patient Characteristics Between DAA and Non- DAA Groups}

Table 1 shows the characteristics of patients in both the DAA and non-DAA groups. The DAA group had a higher frequency of men, alcohol abuse, smoking, and hepatic encephalopathy, and higher values for the MELD score, BMI,
ALT, AST, FIB-4 score, albumin, total bilirubin, eGFR, and PT-INR. By contrast, the non-DAA group had a higher frequency of DM and ascites, as well as greater age and higher values for Child-Pugh score and platelet count. The percentages of patients with HCV genotypes 1, 2,3 , and 4 in the DAA and non-DAA groups were $53.5 \% \quad(n=193)$ and $74.1 \% \quad(n=152)$, $4.4 \% \quad(n=16) \quad$ and $25.9 \% \quad(n=53), \quad 35.7 \%$ $(n=129)$ and $0.0 \%(n=0)$, and $6.4 \%(n=24)$ and $0.0 \%(n=0)$, respectively, indicating a significant difference between the two groups $(p<0.0001)$. The two groups also differed significantly in the proportion of patients who had undergone liver transplantation $(p<0.0001)$. The proportions of individuals with liver-related versus non-liver-related death were $39.6 \%$ and $60.4 \%$ in the DAA group, respectively, and $50.6 \%$ and $19.4 \%$ in the non-DAA group, respectively, indicating no difference between 
the two groups. Causes of liver-related and nonliver-related death are shown in Table 2. Details regarding supportive therapies are shown in Table 3. The calculated propensity scores of patients in the DAA and non-DAA groups were $0.94(0.80-0.99)$ and $0.13(0.05-0.30)$, respectively ( $p<0.0001$, Supplementary Fig. 1$)$.

Table 2 Summary of liver-related and non-liver-related deaths by cause

\begin{tabular}{|c|c|c|}
\hline Cause of death & $\begin{array}{l}\text { Clearance } \\
\text { group } \\
(n=48)\end{array}$ & $\begin{array}{l}\text { No clearance group } \\
(n=156)\end{array}$ \\
\hline $\begin{array}{l}\text { Liver-related } \\
\text { deaths }\end{array}$ & 19 & 79 \\
\hline $\mathrm{HCC}$ & 1 & 37 \\
\hline Liver failure & 17 & 42 \\
\hline $\begin{array}{l}\text { Gastrointestinal } \\
\text { bleeding }\end{array}$ & 1 & 0 \\
\hline $\begin{array}{l}\text { Non-liver-related } \\
\text { deaths }\end{array}$ & 29 & 77 \\
\hline $\begin{array}{l}\text { Malignant } \\
\text { neoplasm }\end{array}$ & $\begin{array}{l}4 \text { lung, } 1 \\
\text { pancreas }\end{array}$ & $\begin{array}{l}4 \text { lung, } 18 \text { digestive } \\
\text { tract, } 3 \text { pancreas, } 2 \\
\text { nonsolid, } 1 \\
\text { thyroid, } 1 \text { ovary, } 1 \\
\text { prostate }\end{array}$ \\
\hline Cardiac disorder & 7 & 14 \\
\hline Infection & $\begin{array}{l}3 \text { pneumonia, } \\
4 \text { sepsis }\end{array}$ & $\begin{array}{l}5 \text { pneumonia, } 6 \\
\text { sepsis, } 1 \text { peritonitis }\end{array}$ \\
\hline $\begin{array}{l}\text { Nervous system } \\
\text { disorder }\end{array}$ & 3 & 6 \\
\hline $\begin{array}{l}\text { Gastrointestinal } \\
\text { disorder }\end{array}$ & 2 & 5 \\
\hline Injury & 2 & 3 \\
\hline Renal disorder & 2 & 3 \\
\hline $\begin{array}{l}\text { Respiratory } \\
\text { disorder }\end{array}$ & 1 & 1 \\
\hline $\begin{array}{l}\text { Vascular } \\
\text { disorder }\end{array}$ & 0 & 3 \\
\hline
\end{tabular}

$D A A$ direct-acting antiviral, $H C C$ hepatocellular carcinoma

\section{Factors Associated with Hepatocarcinogenesis}

Factors associated with hepatocarcinogenesis were analyzed using Cox's proportional hazards model (Table 4). The analyzed factors were age, gender, BMI, alcohol abuse, DM, ascites, hepatic encephalopathy, antiviral therapy, AST, ALT, total bilirubin, albumin, platelet count, PT-INR, and creatinine. High serum albumin (adjusted hazard ratio [aHR] 0.650, 95\% confidential interval [CI] 0.426-0.990, $p=0.0456$ ) and SVR (aHR 0.521, 95\% CI 0.298-0.972, $p=0.0452$ ) were independently associated with hepatocarcinogenesis. Figure $2 \mathrm{a}$ shows that the IPW-adjusted cumulative hepatocarcinogenesis rates in the DAA and non-DAA groups were $0.0 \%$ and $2.3 \%$ at 1 year, respectively, $2.2 \%$ and $7.5 \%$ at 2 years, respectively, and $8.4 \%$ and $12.9 \%$ at 3 years, respectively, indicating a significant difference $(p<0.0001$, IPW-adjusted log-rank test).

\section{Factors Associated with All-Cause Mortality}

Factors associated with all-cause mortality were analyzed using Cox's proportional hazards model (Table 4). The analyzed factors were the same as those for all-cause mortality. High serum albumin (aHR 0.671, 95\% CI $0.517-0.869, p=0.0025)$ and SVR (aHR 0.492, 95\% CI $0.302-0.801, p=0.0044)$ were independently associated with all-cause mortality. Figure $2 \mathrm{~b}$ shows that the IPW-adjusted cumulative all-cause mortality rates in the DAA and non-DAA groups were $5.0 \%$ and $16.2 \%$ at 1 year, respectively, $12.3 \%$ and $23.6 \%$ at 2 years, respectively, and $17.6 \%$ and $34.2 \%$ at 3 years, respectively, indicating a significant difference $(p<0.0001$, IPW-adjusted log-rank test).

\section{Factors Associated with Liver-Related Mortality}

Table 4 shows the factors associated with liverrelated mortality according to Fine and Gray proportional hazards models. The analyzed factors were the same as those for all-cause 
Table 3 Supportive therapies for decompensated cirrhosis

\begin{tabular}{lllr}
\hline & DAA group $(\boldsymbol{n}=\mathbf{3 6 4})$ & Non-DAA group $(\boldsymbol{n}=\mathbf{2 4 9})$ & $\boldsymbol{p}$ \\
\hline Diuretics & $177(48.6)$ & $146(58.6)$ & 0.017 \\
Lactulose or lactitol & $71(19.5)$ & $58(23.3)$ & 0.268 \\
Antibiotics & $31(8.5)$ & $32(12.8)$ & 0.104 \\
BCAA & $0(0.0)$ & $77(30.9)$ & $<0.001$ \\
Beta-blockers & $108(26.7)$ & $9(3.6)$ & $<0.001$ \\
Blood products & $14(3.8)$ & $76(30.5)$ & $<0.001$ \\
\hline
\end{tabular}

$D A A$ direct-acting antiviral, $B C A A$ branched-chain amino acid

Table 4 Factors associated with hepatocarcinogenesis and all-cause, liver-related, and non-liver-related mortality

\begin{tabular}{|c|c|c|c|}
\hline & Hazard ratio & $95 \%$ confidence interval & $p$ values \\
\hline \multicolumn{4}{|c|}{ Factors associated with hepatocarcinogenesis } \\
\hline Albumin $(\mathrm{g} / \mathrm{dL})$ & 0.650 & $0.426-0.990$ & 0.0456 \\
\hline SVR & 0.521 & $0.298-0.972$ & 0.0452 \\
\hline \multicolumn{4}{|c|}{ Factors associated with all-cause mortality } \\
\hline Albumin (g/dL) & 0.671 & $0.517-0.869$ & 0.0025 \\
\hline SVR & 0.492 & $0.302-0.801$ & 0.0044 \\
\hline \multicolumn{4}{|c|}{ Factors associated with liver-related mortality } \\
\hline Albumin (g/dL) & 0.503 & $0.355-0.712$ & 0.0001 \\
\hline Creatinine $(\mathrm{mg} / \mathrm{dL})$ & 0.641 & $0.482-0.822$ & 0.0022 \\
\hline SVR & 0.326 & $0.143-0.743$ & 0.0077 \\
\hline \multicolumn{4}{|c|}{ Factors associated with non-liver-related mortality } \\
\hline Creatinine $(\mathrm{mg} / \mathrm{dL})$ & 1.167 & $1.047-1.301$ & 0.0051 \\
\hline SVR & 0.520 & $0.326-0.829$ & 0.0060 \\
\hline
\end{tabular}

SVR sustained virological response

mortality. Multivariate analysis showed that high serum albumin (aHR 0.503, 95\% CI $0.355-0.712, p=0.0001)$, high serum creatinine (aHR 0.641, 95\% CI 0.482-0.822, $p=0.0022$ ), and SVR (aHR 0.326, 95\% CI 0.143-0.743, $p=0.0077)$ were independently associated with liver-related mortality. Figure $2 \mathrm{c}$ shows that the IPW-adjusted cumulative incidence rates of liver-related mortality with and without DAA therapy were $2.6 \%$ and $7.5 \%$ at 1 year, respectively, $6.2 \%$ and $10.5 \%$ at 2 years, respectively, and $7.4 \%$ and $18.0 \%$ at 3 years, respectively, indicating a significant difference between the two groups $(p<0.0001$, IPW-adjusted log-rank test). 

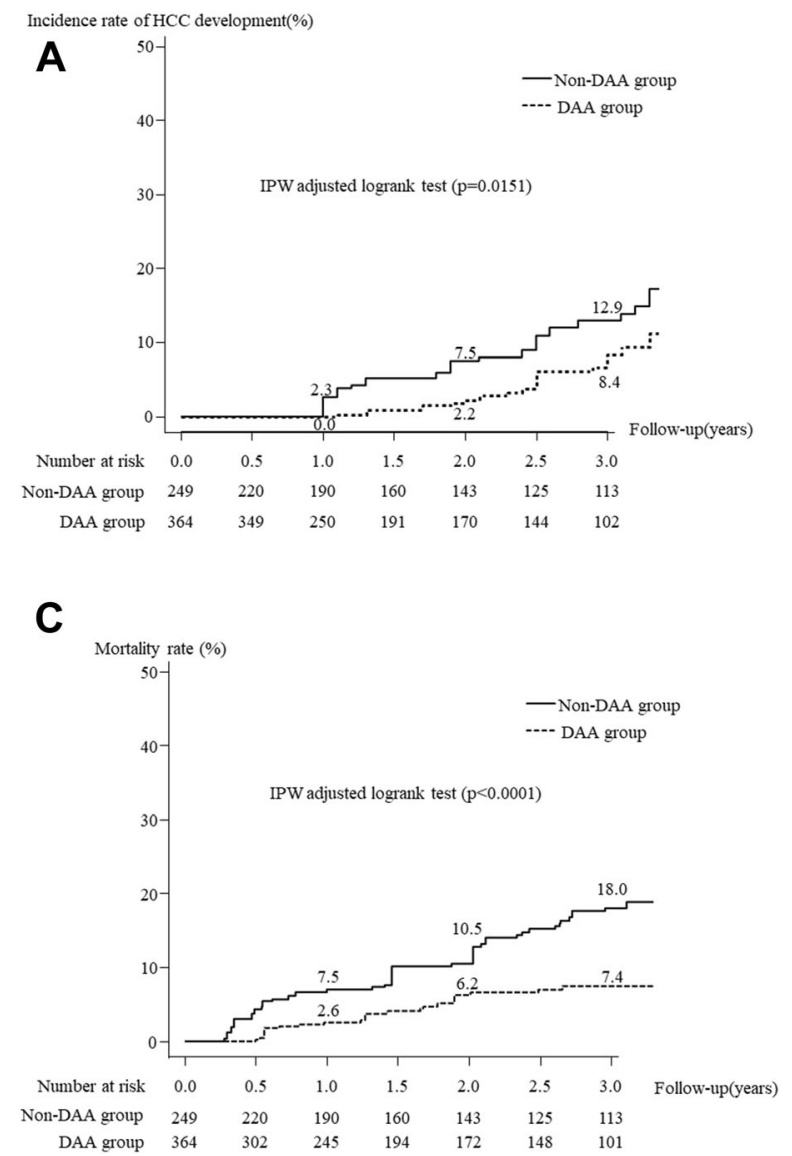

Fig. 2 Rates of hepatocarcinogenesis and all-cause, liverrelated, and non-liver-related mortality with and without DAA therapy and adjusted by IPW. a The cumulative hepatocarcinogenesis rates in the DAA and non-DAA groups were $0.0 \%$ and $2.3 \%$ at 1 year, respectively, $2.2 \%$ and $7.5 \%$ at 2 years, respectively, and $8.4 \%$ and $12.9 \%$ at 3 years, respectively, indicating a significant difference between the two groups $(p<0.0001$, IPW-adjusted $\log$ rank test). $\mathbf{b}$ The cumulative all-cause mortality rates in the DAA and non-DAA groups were $5.0 \%$ and $16.2 \%$ at 1 year, respectively, $12.3 \%$ and $23.6 \%$ at 2 years, respectively, and $17.6 \%$ and $34.2 \%$ at 3 years, respectively, indicating a significant difference between the two groups $(p<0.0001$, IPW-adjusted log-rank test). c The

\section{Factors Associated with Non-Liver-Related Mortality}

Table 4 shows the factors associated with nonliver-related mortality according to Fine and Gray proportional hazards models. The analyzed factors were the same as those for all-cause
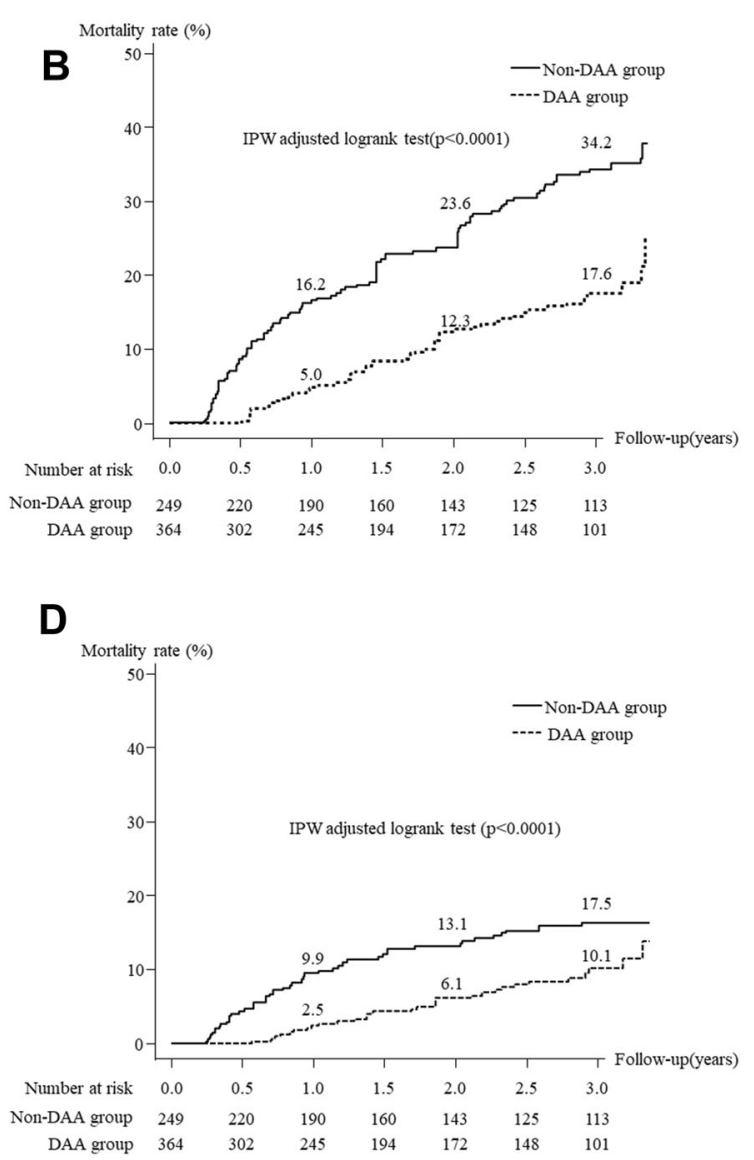

cumulative incidence rates of liver-related mortality with and without DAA therapy were $2.6 \%$ and $7.5 \%$ at 1 year, respectively, $6.2 \%$ and $10.5 \%$ at 2 years, respectively, and $7.4 \%$ and $18.0 \%$ at 3 years, respectively, indicating a significant difference between the two groups $(p<0.0001$, IPW-adjusted log-rank test). $\mathbf{d}$ The cumulative incidence rates of non-liver-related mortality with and without DAA therapy were $2.5 \%$ and $9.9 \%$ at 1 year, respectively, $6.1 \%$ and $13.1 \%$ at 2 years, respectively, and $10.1 \%$ and $17.5 \%$ at 3 years, respectively, indicating a significant difference between the two groups $(p<0.0001$, IPW-adjusted logrank test). DAA direct-acting antiviral, IPW inverse probability weighting, SVR sustained virological response

mortality. Multivariate analysis showed that high creatinine (aHR, 1.167, 95\% CI, 1.047-1.301, $p=0.0051$ ) and SVR (aHR, 0.520, $95 \% \mathrm{CI}, 0.326-0.829, p=0.0060$ ) were independently associated with non-liver-related mortality. Figure $2 \mathrm{~d}$ shows that the IPW-adjusted cumulative incidence rates of non-liver- 
related mortality with and without DAA therapy were $2.5 \%$ and $9.9 \%$ at 1 year, respectively, $6.1 \%$ and $13.1 \%$ at 2 years, respectively, and $10.1 \%$ and $17.5 \%$ at 3 years, respectively, indicating a significant difference between the two groups ( $p<0.0001$, IPW-adjusted log-rank test).

\section{DISCUSSION}

The advent of highly effective DAA drugs has transformed the treatment options for HCV-infected patients with DC $[11,12]$. In this study, we examined the results of a 12-week course of antiviral therapy in a large, heterogeneous group of patients with DC or life-threatening complications of HCV infection in the UK (the HCV Research UK registry), and compared the outcomes of this group, who received DAAs and achieved SVR, with a group of patients in Japan who were not treated with DAAs. We found that after adjustment for potential confounding factors, viral eradication reduced not only liverrelated mortality but also non-liver-related mortality in patients with $\mathrm{HCV}$ infection and DC.

Hepatocarcinogenesis was associated with low serum albumin and no viral eradication. Calvaruso et al. reported that patients treated with DAA therapy had a lower incidence of HCC if they achieved SVR than if they did not [23]. All-cause mortality in this population was associated with low serum albumin and no viral eradication. There has previously been little information on how liver-related and non-liverrelated mortality are affected by viral eradication in patients with HCV infection and DC. This study showed that in these patients, liverrelated mortality was associated with low serum albumin, high serum creatinine, and no viral eradication. Prognosis was closely related to $\mathrm{HCV}$ clearance, and the HR of the DAA group was about one-fourth of that in the non-DAA group. It is clear that eradicating HCV is very important to reduce liver-related mortality. Impaired liver function, as indicated by albumin level, was associated with increased liverrelated mortality. Judging from these results, it is considered that antiviral therapy should be started as early as possible during the period of mild liver injury. On the other hand, in this study, non-liver-related mortality was associated with low serum creatinine and no viral eradication. Many previous studies involving long-term follow-up of individuals receiving IFN-based therapy showed that viral eradication reduced not only liver-related mortality but also non-liver-related mortality in patients with chronic hepatitis and compensated cirrhosis [7-9]. Our study is the first to show that viral eradication reduces non-liver-related mortality even in patients with HCV infection and DC. $\mathrm{HCV}$ infection has been recognized as a systematic disease with both hepatic and extrahepatic manifestations [24, 25]. Chronic HCV infection is associated with pathophysiological changes outside the liver, including those involving the metabolic, cardiovascular, and neurological systems, as well as autoimmune and immune-mediated conditions such as mixed cryoglobulinemia, thyroid disease, and glomerulonephritis. Studies have also shown that treatment to eradicate $\mathrm{HCV}$ infection may improve some extrahepatic manifestations of $\mathrm{HCV}$ independently of the severity of underlying liver disease $[26,27]$. In this study, low serum creatinine was associated with increased mortality related to liver disease, and high serum creatinine was associated with increased mortality caused by non-liver-related disease. We consider this result to be a reciprocal change. It is presumed that extrahepatic disease such as renal dysfunction worsened before the progression to liver failure, resulting in deaths unrelated to liver disease [28].

The main limitation of this study was the difference in characteristics between the treated and control subjects. Untreated Japanese patients with DC were selected on the basis of the same criteria as treated patients in the UK. While it would have been preferable to conduct a randomized controlled trial of treatment versus no treatment, this would have been unethical. There were significant differences in the background factors of the DAA group in the UK and the non-DAA group in Japan. Patients in Japan were an average of 16 years older and were more likely to be men and to have DM than those in the UK. In contrast, patients in the UK had a higher BMI and higher rates of 
alcohol abuse and smoking than those in Japan. Genotype 3 was present in the UK, but not in Japan. Liver transplantation is uncommon in Japan because most patients are elderly and many Japanese tend to refuse organ donation by themselves or their relatives because of religious and cultural issues. By contrast, approximately $10 \%$ of patients received a liver transplant in the UK. However, there was no difference between the two groups in Child-Pugh class, ALBI score, or ALBI grade, all of which were used as indicators of liver function. The FIB-4 score, used as a marker of fibrosis, was higher in the UK than in Japan. In order to reduce the confounding effects of covariates, we used propensity score matching based on age, gender, BMI, alcohol abuse (presence or absence), DM (presence or absence), ascites (presence or absence), hepatic encephalopathy (presence or absence), AST, ALT, total bilirubin, albumin, platelet count, PT-INR, and creatinine. We then applied IPW to Kaplan-Meier analysis of hepatocarcinogenesis and all-cause mortality to adjust for potential imbalances between the DAA and non-DAA groups [20]. In addition, IPW cumulative incidence functions were calculated to appropriately account for competing events, including liver-related and non-liverrelated mortality [21]. The results of the analysis demonstrated that the DAA group survived longer than the non-DAA group despite their different background factors. However, the findings of the propensity score analyses might have been limited by biases related to unmeasured and hidden covariates (e.g., mental illness and risky drug behaviors). A second limitation is the presence of racial differences. The rates of chronic HCV infection, response to treatment, and development of complications differ among different racial and ethnic groups. African Americans demonstrated a lower rate of SVR to DAA treatment for HCV infection [29]. In addition, African Americans and Asians with HCV were shown to have a two-fold and fourfold increased risk, respectively, of developing HCC when compared to Caucasians [30]. That study did not compensate for differences in race. No host or viral genetic differences have yet been identified to explain the racial disparities in the incidence of chronic $\mathrm{HCV}$, response to treatment, or development of HCC. In our study, we were unable to correct for racial and ethnic differences. A third limitation of our study is that the duration of follow-up was short, making it impossible to assess long-term outcomes associated with DAAs. Nevertheless, an inverse relation was noted between treatment with DAAs and both liver-related and non-liver-related mortality in patients with DC over this short-term follow-up period, and a longer duration of follow-up would probably not change these findings.

There were some patients with compensated cirrhosis in both groups. Specifically, $13.7 \%$ of patients in the UK (DAA group) and $14.5 \%$ of patients in Japan (non-DAA group) were Child-Pugh class A at baseline, but had past decompensation events.

\section{CONCLUSION}

SVR obtained by DAA therapy for DC prolongs survival. Moreover, this effect applies not only to liver-related mortality but also to non-liverrelated mortality.

\section{ACKNOWLEDGEMENTS}

We thank the participants of the study.

Funding. This work was supported by Health and Labour Sciences Research Grants (Research on Hepatitis) from the Ministry of Health, Labour and Welfare of Japan. HCV Research UK was established by a grant from the Medical Research Foundation (award no. C0365). The Rapid Service Fee was funded by the authors.

Authorship. All named authors meet the International Committee of Medical Journal Editors (ICMJE) criteria for authorship for this article, take responsibility for the integrity of the work as a whole, and have given their approval for this version to be published.

Authorship Contributions. Concept and study design: Takashi Kumada and William L. 
Irving. Data acquisition: all authors. Analyses of the data: Junko Tanaka and Toshifumi Tada. Statistics: Junko Tanaka. Supervision: Kazuaki Chayama, Philip J. Johnson, and William L. Irving. Manuscript preparation: Takashi Kumada and William L. Irving. Review and approval: all authors.

Disclosures. Takashi Kumada (Gilead Sciences, AbbVie), Hidenori Toyoda (Gilead Sciences, AbbVie, Merck Sharp and Dohme, Bayer), Junko Tanaka (Gilead Sciences, AbbVie), Kazuaki Chayama (Gilead Sciences, Merck Sharp and Dohme, AbbVie, Ajinomoto, BristolMyers Squibb, Chugai, Sumitomo Dainippon, Mitsubishi Tanabe, Abbot, Astellas, Otsuka, Roche, Janssen, EA, and Toray) and William L. Irving (Roche, Janssen Cilag, Gilead Sciences, Novartis, Boehringer Ingelheim, Merck Sharp and Dohme, GlaxoSmithKline, Pfizer, BristolMyers Squibb). Satoshi Yasuda, Toshifumi Tada and Philip J. Johnson have nothing to disclose.

Compliance with Ethics Guidelines. Ethics approval for HCV Research UK was given by the National Research Ethics Service (NRES) Committee East Midlands-Derby 1 (Research Ethics Committee reference 11/EM/0314), and the proposal for the analysis reported herein was accepted by the HCV Research UK Tissue and Data Access committee in January 2019 (Request No. TR000444). Informed consent was obtained from each patient included in the UK group. The requirement for consent to participate in the Japanese group was waived by the ethics committee because of the retrospective nature of the study. The study protocol was also approved by the institutional review board of Ogaki Municipal Hospital in March 2019 and was conducted in compliance with the Helsinki Declaration.

Data Availability. The datasets are available from the corresponding author on reasonable request.

Open Access. This article is licensed under a Creative Commons Attribution-NonCommercial 4.0 International License, which permits any non-commercial use, sharing, adaptation, distribution and reproduction in any medium or format, as long as you give appropriate credit to the original author(s) and the source, provide a link to the Creative Commons licence, and indicate if changes were made. The images or other third party material in this article are included in the article's Creative Commons licence, unless indicated otherwise in a credit line to the material. If material is not included in the article's Creative Commons licence and your intended use is not permitted by statutory regulation or exceeds the permitted use, you will need to obtain permission directly from the copyright holder. To view a copy of this licence, visit http://creativecommons.org/licenses/by$\mathrm{nc} / 4.0 /$.

\section{REFERENCES}

1. Westbrook RH, Dusheiko G. Natural history of hepatitis C. J Hepatol. 2014;61(1 Suppl):S58-68.

2. McDonald SA, Innes HA, Aspinall E, et al. Prognosis of 1169 hepatitis C chronically infected patients with decompensated cirrhosis in the predirect-acting antiviral era. J Viral Hepat. 2017;24:295-303.

3. Carrión JA, Martínez-Bauer E, Crespo G, et al. Antiviral therapy increases the risk of bacterial infections in HCV-infected cirrhotic patients awaiting liver transplantation: a retrospective study. J Hepatol. 2009;50:719-28.

4. Iacobellis A, Siciliano M, Perri F, et al. Peginterferon alfa-2b and ribavirin in patients with hepatitis $\mathrm{C}$ virus and decompensated cirrhosis: a controlled study. J Hepatol. 2007;46:206-12.

5. Afdhal N, Zeuzem S, Kwo P, et al. Ledipasvir and sofosbuvir for untreated HCV genotype 1 infection. N Engl J Med. 2014;370:1889-98.

6. Lawitz E, Sulkowski MS, Ghalib R, et al. Simeprevir plus sofosbuvir, with or without ribavirin, to treat chronic infection with hepatitis $\mathrm{C}$ virus genotype 1 in non-responders to pegylated interferon and ribavirin and treatment-naïve patients: the COSMOS randomised study. Lancet. 2014;384:1756-65.

7. van der Meer AJ, Veldt BJ, Feld JJ, et al. Association between sustained virological response and allcause mortality among patients with chronic hepatitis $C$ and advanced hepatic fibrosis. JAMA. 2012;308:2584-93. 
8. Tada $\mathrm{T}$, Kumada $\mathrm{T}$, Toyoda $\mathrm{H}$, et al. Viral eradication reduces all-cause mortality in patients with chronic hepatitis $C$ virus infection: a propensity score analysis. Liver Int. 2016;36:817-26.

9. Nahon P, Bourcier V, Layese R, et al. Eradication of hepatitis $C$ virus infection in patients with cirrhosis reduces risk of liver and non-liver complications. Gastroenterology. 2017;152:142-56.

10. Charlton M, Everson GT, Flamm SL, et al. Ledipasvir and sofosbuvir plus ribavirin for treatment of HCV infection in patients with advanced liver disease. Gastroenterology. 2015;149:649-59.

11. Curry MP, O'Leary JG, Bzowej N, et al. Sofosbuvir and velpatasvir for HCV in patients with decompensated cirrhosis. N Engl J Med. 2015;373: 2618-28.

12. Foster GR, Irving WL, Cheung MC, et al. Impact of direct acting antiviral therapy in patients with chronic hepatitis $\mathrm{C}$ and decompensated cirrhosis. J Hepatol. 2016;64:1224-31.

13. Cheung MCM, Walker AJ, Hudson BE, et al. Outcomes after successful direct-acting antiviral therapy for patients with chronic hepatitis $C$ and decompensated cirrhosis. J Hepatol. 2016;65:741-7.

14. Matsuo S, Imai E, Horio MY, et al. Revised equations for estimated GFR from serum creatinine in Japan. Am J Kidney Dis. 2009;53:982-92.

15. Sterling RK, Lissen E, Clumeck N, et al. Development of a simple noninvasive index to predict significant fibrosis in patients with HIV/HCV coinfection. Hepatology. 2006;43:1317-25.

16. Vallet-Pichard A, Mallet V, Nalpas B, et al. FIB-4: an inexpensive and accurate marker of fibrosis in $\mathrm{HCV}$ infection. Comparison with liver biopsy and fibrotest. Hepatology. 2007;46:32-6.

17. Johnson PJ, Berhane S, Kagebayashi C, et al. Assessment of liver function in patients with hepatocellular carcinoma: a new evidence-based approach-the ALBI grade. J Clin Oncol. 2015;33: 550-8.

18. American Diabetes Association. Diagnosis and classification of diabetes mellitus. Diabetes Care. 2010;33:S62-9.

19. Fine JP, Gray RJ. A proportional hazards model for the subdistribution of a competing risk. JASA. 1999;94:496-509.
20. Austin PC, Stuart EA. Moving towards best practice when using inverse probability of treatment weighting (IPTW) using the propensity score to estimate causal treatment effects in observational studies. Version 2. Stat Med. 2015;34:3661-79.

21. Bolch CA, Chu H, Jarosek S, Cole SR, Elliott S, Virnig B. Inverse probability of treatment-weighted competing risks analysis: an application on longterm risk of urinary adverse events after prostate cancer treatments. BMC Med Res Methodol. 2017; 17:93.

22. Kanda Y. Investigation of the freely available easyto-use software 'EZR' for medical statistics. Bone Marrow Transplant. 2013;48:452-8.

23. Calvaruso V, Cabibbo G, Cacciola I, et al. Incidence of hepatocellular carcinoma in patients with HCVassociated cirrhosis treated with direct-acting antiviral agents. Gastroenterology. 2018;155: 411-21.

24. Negro F, Forton D, Craxì A, et al. Extrahepatic morbidity and mortality of chronic hepatitis C. Gastroenterology. 2015;149:1345-60.

25. Younossi Z, Park H, Henry L, et al. Extrahepatic manifestations of hepatitis C: a meta-analysis of prevalence, quality of life, and economic burden. Gastroenterology. 2016;150:1599-608.

26. van der Meer AJ, Feld JJ, Hofer H, et al. Risk of cirrhosis-related complications in patients with advanced fibrosis following hepatitis $\mathrm{C}$ virus eradication. J Hepatol. 2017;66:485-93.

27. Ioannou GN, Beste LA, Green PK, et al. Increased risk for hepatocellular carcinoma persists up to 10 years after HCV eradication in patients with baseline cirrhosis or high FIB-4 scores. Gastroenterology. 2019;157:1264-78.

28. Khan MU, Mahmoud MI, Butt AA. Hepatitis C virus and chronic kidney disease. Expert Rev Gastroenterol Hepatol. 2020;14:1-11.

29. Kanwal F, Kramer JR, El-Serag HB, et al. Race and gender differences in the use of direct acting antiviral agents for hepatitis $C$ virus. Clin Infect Dis. 2016;63:291-9.

30. Nguyen MH, Whittemore AS, Garcia RT, et al. Role of ethnicity in risk for hepatocellular carcinoma in patients with chronic hepatitis $\mathrm{C}$ and cirrhosis. Clin Gastroenterol Hepatol. 2004;2:820-4. 